\title{
Valéry mythoclaste
}

\section{Françoise Haffner}

Université Paul Valéry, Montpellier, Francia

L'œuvre de Valéry est difficile à saisir puisqu'elle ne s'articule pas en une forme ou un discours unifiée - qui permettrait une saisie synthétique, claire sur un plan rationnel - ni en une cohérence "mythique" que conforterait la signature de l'auteur et qui permettrait alors de la poser pour bâtir la figure exemplaire du Poète ou de l'Intellectuel. Cela a été fait - il y a longtemps, par certaines critiques et lecteurs figeant le Poète du Cimetière Marin et de la Jeune Parque en la Figure d'un praticien de la Poésie Pure, héritier dévoyé - parce que perçu comme néo-classique - de son maître Mallarmé ; par d'autres qui en ont fait l'incarnation del'Intellectuel Bourgeois, intellectuel un peu désincarné, se voulant au-dessus de la mélée, et pour tout dire réactionnaire ; ou bien, autre attitude, les lecteurs pressés se sont hâtés d'utiliser le sens du raccourci de Valéry, son écriture fragmentée, pour l'enfermer en quelques "nombreuses" formules aphoristiques, à inscrire dans un dictionnaire de citations classées par thèmes, permettant ainsi de placer telle phrase "définitive" à propos de l'Art, de la Civilisation, de l'Histoire, de l'Europe, de la Crise de l'Esprit... et même des Idoles, des Idées-Perroquets comme il les appelait.

Pourtant, depuis la publication des Cahiers dans l'Édition du CNRS entreprise entre 1957 et 1961, à laquelle il faut ajouter l'accès à une masse de manuscrits (ceux des Cahiers, mais aussi ceux des brouillons) déposée à la Bibliothèque Nationale -, les chercheurs valéryens ont entrepris la réévaluation de cette œuvre. Quant aux lecteurs "anonymes", les Euvres et l'Anthologie des Cahiers parues 
à la Bibliothèque de la Pléiade, l'entreprise en cours de l'édition typographique des Cahiers écrits entre 1894 et 1914 chez Gallimard peuvent leur permettre de saisir l'ampleur de la démarche, et l'inanité de toute réduction à un ćpithète. C'est pourtant ce que le titre de ma communication semble faire - et fait peut-être... s'inscrivant dans une liste où vous pourrez trouver Valéry néo-positiviste, Valéry nietzschéen, Valéry sémioticien, structuraliste, mystique sans Dieu, apophatique etc.

Effets de lecture?

Valéry inscrit dans son œuvre l'espace d'un débat avec le lecteur - soutendu par une théorie de l'auteur dépouillée de toute transcendance, et laissant la place au pouvoir du lecteur. Du coup, le nom de l'auteur n'a plus de sens et il peut dire dans Notre destin et les Lettres: "Je porte le nom que vous voudrez, et c'est mon véritable nom." (E. T. II, 1064).

Pourtant le choix de mythoclaste pour caractériser Valéry n'est pas seulement le signe de mon pouvoir de lecteur. Il est inscrit dans la lettre même de l'œuvre, synthétisant un projet - compris non pas comme intention à réaliser dans une forme, mais comme démarche même.

Je tiens à souligner que la question du Je et du Moi est essentielle dans l'entreprise mythoclaste - ou Idoloclaste ("L'idoloclaste", c'est ainsi que Valéry se "nomme" parfois) - et qu'elle met en cause la question de la personne, de l'être social composé d'héritages plus ou moins conscients / inconscients.

Dans un des Cahiers de 1943 (C. XXVII, 374-375, C.I. 219, manuscrit B.N Cahier 240 - F. 57 v.), sous le titre "Ego", il fait un bilan sur son Moi avec une distance ironique par rapport à l'adhésion à l'image de lui-même que les autres lui renvoient (c'est-à-dire le sentiment, en cette circonstance d'une certaine importance) et il conclut la passage :

Bref, sum qui sum, et il faut tâcher de ne pas se laisser être quem me faciunt. 
La question est de surmonter la Personne, qui est une Idole.

Et j'ai déclaré la guerre aux Idoles, il y a 50 ans passés !

Mais elles sont trop ! Le corps, la Vie, qui est la Mort, l'Amour, etc.

Enfin, tout ce qui ne se laisse pas réduire à l'égalité devant le $M o i$ pur et au possible.

Un tel constat marque à la fois l'ambition de Valéry et la conscience de son échec. En effet, la guerre aux Idoles est le revers de la poursuite d'une connaissance véritable amenant la compréhension - la guerre aux Idoles fait partie d'un véritable protocole épistémologique qui s'établit après la crise de 1892 et qui aboutira à la mise en place de ce qu'il appelle mon "système" (avec les guillemets). Il reprend, mais avec une détermination sans faille la table rase cartésienne.

De la conscience du "connu d'avance", il passe à une Révolution plus radicale qu'il articulera sur une réflexion sur le (ou plutôt les) langage(s) à partir de l'expérience de ceux-ci - et ce qui est essentiel dans cette expérience, comme le note Simon Lantiéri dans Paul Valéry, néo-positiviste ?, "celle de l'engagement des mots dans la production d'une connaissance ou d'une jouissance".

Valéry démonte remarquablement cela dans un passage des Cahiers (1939 - CNRS - XXII - 199-204) :

C'est mon "système" !/ - qui conduit à refuser toute valeur "vraie" - toute valeur acceptable in intimo corde = aux évaluations toutes reçues et jusqu' aux mots $=$ je considérai comme extérieures et provisoires, simples objets ou moyens de troc toutes notions - et entendis affecter à chacune, sa définition Absolue c'est-à-dire ce qu'elle amenait réellement à l'esprit à telle occasion. Ce décret était essentiel - à ma Révolution - Nihil est in verbis quod non fuerit responsia tua - et nihil majus. C'était l'observation pure. 
Ici, Valéry se met délibérément dans la lignée des empiristes, des nominalistes, de Protagoras à Condillac en passant par Epicure, Montaigne, Gassendi ou Locke. Inutile de souligner que Valéry - dont on a fait un peu trop vite le chantre de l'Intellect pur, en oubliant que ce dernier s'inscrivait dans les pouvoirs de l'Esprit - se revendique donc comme matérialiste. Il joue avec la "formule" célèbre : Nihil est in intellecti quod fueri in sensu (Il n'y a rien dans l'intellect qui n'ait été d'abord dans la sensation).

Mais il est intéressant de voir ce qu'il transforme dans la formule : le "in intellecti" devient "in verbis" : dans les mots - "quod non fuerit responsia tua" - qui n'ait été ta réponse. Qu'est-ce à dire ? Qu'il s'agit de mettre en rapport les mots (les verba) avec "l'intime cœur" - le dedans. Mais un dedans qui passe au crible toutes les notions. Se met donc en place une vaste entreprise critique fondée sur l'observation pure - autant que faire se peut - de ce qui se passe dans l'esprit appréhendant les mots.

Le travail poétique, "la cuisine verbale de la poésie" lui faisait bien voir que :

i nous pouvons combiner les mots, c'est qu'ils ne sont pas... des choses. Ainsi, penser (au sens actif), c'est à la fois tenir les sens demots pour choses, pour non-mots - et cependant les combiner comme des jetons libres, ou modeler les images adjacentes comme couleurs ou lignes ou glaise.

D'où résulteraient : $1 \%$ des libertés, $2 \%$ des contraintes tout autres que celles de l'usage ordinaire des mots et des idées.) (ibid. C.I. 167-168 - C. XXII, 202-203-1939)

Valéry reprend donc le problème de l'arbitraire du langage, renouant avec une philosophie qu' on peut qualifier de classique, mais en poursuivant son dessein de telle sorte qu'il va rencontrer, croiser un certain nombre de réflexions de la modernité sur le langage et plus généralement, les signes portés par le renouvellement de la logique 
(Wittgenstein, le scepticisme anglo-saxon), de la linguistique (Saussure, mais plus sûrement Benveniste), de la sémiotique (Pierce et Eco), de la réflexion esthétique (de l'école de Francfort et plus particulièrement d'Adorno). Je pense aussi que le travail de Valéry - ce que j'appellerai son décapage critique - devrait intéresser la réflexion socio-critique dans la mesure où sa critique de ce qu'il appelle la Fiducia est la mise-en-relation du langage (et du socio-culturel au sens le plus large) et du sujet. Ce travail de croisement avec la socio-critique d'une part, Adorno d'autre part est en cours ou plutôt à l'état de gestation. Pour aujourd'hui, je me contenterai de poser quelques "nœuds" de la pensée valéryenne qui me paraissent plus particulièrement intéressants à croiser avec la réflexion socio-critique.

Peut-on définir rapidement le système valéryen? Il ne s'agit pas d'un système explicatif fermé - se donnant comme vision du monde car ce serait un nouveau monstre, un de ces mythes que Valéry s'est décidé à pourfendre. Élaborant son système sur la relation C.E.M. (Corps-Esprit-Monde), Valéry essaie d'être au plus près (conscience de conscience) dans sa relation avec $\mathrm{C}$ et $\mathrm{M}$.

Il essaie de mettreen place un système fonctionneletopérationnel pour un "Moi". Ce qui enlève toute connotation "universelle" à la notion de système.

Ego. Mon "système ?" - C'est Moi. Mais moi - en tant qu'un moi est convergence, et variations.

Sans quoi, ce système ne serait qu' un système entr'autres que je pourrais faire. Mais cette diversité est précisément Moi. Je suis cette diversité possible.

Comment peut-on faire un système, - un édifice d'idées qui ne soit pas à la merci d'une idée ? (Cahier 240 - F. 57 v. manuscrit BN)

Valéry découvre le change, le discontinu, l'éclatement de la personne au profit d'un Moi qui se dédouble : 
Dans le réfléchi JE-ME. Je est le Moi pur. ME ou MOI la personne. Quelle relation entre ces deux termes? Noterque $M O I$ (ME) peut être substitué par n'importe quel autre. Mais que JE n'est pas substituable. C'est une direction, une origine, un infra-tout, un fonctionnement in.

JE n'est pas du tout un Pro-nom - car il n'y a pas de nom en moi pour moi, car il n'y a pas d'individu, d'objet pour ce qui est produit par tout objet comme il repousse chaque objet. (Cahier 240 - F. 51).

$J E$ point de référence car origine de l'énonciation, uniquement repérable dans les variations successives qui sont les réponses à tout objet.

Le langage peut-il pleinement rendre compte de cette relation au Monde ?

A mesure que l'on s'approche du réel, on perd la parole. Un objet n'est exprimable que par un nom plus grand que lui et qui n'est que le signe de sa multiplicité de transformations implicites - ou bien par métaphores ou bien par constructions. Le réel est intransformable (C. II. 554 - 1901-1902).

Et donc intraduisible.

L'expérience vécue est, elle aussi, intraduisible dans sa totalité. La plus grande partie d'un discours ne permet pas d'être retraduit dans le non-discours qui était son modèle. A partir de là - de cet arbitraire du langage - toutes les manipulations sont possibles : conscientes, inconscientes -ou non-conscientes - manipulations d'autrui et auto-manipulation.

Quel paradoxe que l'art de manier les choses par des signes qui leur sont extérieurs et étrangers ! et dont la correspondance même avec elles est tout arbitraire ! Il faut que chaque signe soit 
doublée d'un fantôme où s'attache le signe, autre fantôme. Les signes combinés, combinent les fantômes - et une machine spéciale permet de repasser des fantômes aux choses - et de leur imposer, aux choses, le même sort que les faciles fantômes ont enduré dans le lieu bizarres où ils sont esclaves des signes (C. III, 734 - 1905-1906).

Dans le dialogue de soi à soi qu'est l'entreprise des Cahiers Valéry reprend le dialogue entre Hermogène et Cratyle dans le Cratyle de Platon. Hermogène défend la thèse de la valeur fiduciaire (c'est-à-dire fondée sur la croyance en une valeur arbitraire à laquelle on fait crédit) alors que pour Cratyle, le langage a une valeur mimétique, c'est-à-dire qu'il participe de la même substance que le réel avec lequel il entretient des liens analogiques. Pour Socrate et Platon, le verbe, le langage, n'a ni valeur fiduciaire, ni valeur mimétique mais est rapport possible avec la "réalité" par une forme déterminée de la logique, permettant d'ailleurs, par ce type de langage, la médiation entre le monde "concret", copie de la "vraie réalité" et le monde des Idées, seul Réel.

Se mettant, comme nous l'avons vu, du côté du nominalisme, Valéry ne va pas s'installer dans un scepticisme généralisé qui enleverait tout pouvoir à l'homme.

Sa recherche est en fait portée par la question du possible qui apparaît de multiples façons dans son œuvre, mais qui est presque toujours ouverte par le "que peut... ?"

Dans sa lutte contre le fiduciaire, Valéry va entreprendre une sorte de tératologie du langage, ou mieux des systèmes de signes, qui structurent les ensembles socio-culturels - et évidemment leur production. Le résultat d'une telle entreprise exile "les mots sans fond" : "C'était fini de la métaphysique - de la mystique - de la société - de la partie de poésie et surtout de l'histoire et du roman, croyances !... Tout ce fiduciaire s'évanouit".

Il s'agissait bien pour lui de "crever la Fiducia", qui fabrique du "pseudo-réel" en lui donnant sens - alors que la réalité en soi n’a pas 
de signification. La Fiducia est le domaine des signes qui s'organisent en fiction leurrante, selon une réorganisation de la temporalité où par exemple la concaténation fait figure de relation de causalité, où hétérogène peut se traduire en homogène - triomphe donc de l'Illusion aliénante qu' on trouve particulièrement à l'œu vre pour Valéry dans la Théologie, l'Histoire, la Philosophieet le Roman. Mimésis de l'Illusion creée par l'Imaginaire.

Pour "crever la Fiducia" il se sert de formules provocantes, à connotations joyeusement blasphématoires, qui entent son propos sur ceuxdes philosophes du XVIII ${ }^{\mathrm{me}}$ siècle(et particulièrement Diderot): "In principio erat Verbum - Au début était la fable. Au début était la blague. Mais nous ne sommes plus au commencement" (C. IV - 204), et encore dans Tel quel. Autres Rhumbs (E. T. II. 694) :

Au commencement était la blague. Et en effet toutes les histoires s'approfondissent en Fables.

Tout commence invariablement par des contes. La Genèse, l'exposition du système du monde : naissances dans un chou.

Mais comment les puissances de l'illusion fonctionnent-elles ? Comment Valéry est-il arrivé à les déjouer?

Dans le Cahier 240 au feuillet 35, recto, Valéry, faisant le bilan de sa "méthode", souligne qu'il s'agissait de prendre conscience du niveau de fonctionnement de chaque élément, de ne pas établir des connections illusoires :

Ma grande découverte de 1892. Ce qui est mental est mental. Ce qui est verbal est verbal. C'est-à-dire soumis à telles conditions fonctionnelles ses substitutions, d'excitabilité, de durée etc... qui n'ont aucun rapport avec leur figure.

Alors que dans le fonctionnement "ordinaire", il y a changement de plans - tour de passe-passe - instaurant ainsi tout système de croyances. 
Toujours dans le même feuillet, mais au verso, Valéry analyse ainsi le fonctionnement du fiduciaire :

La croyance à $\mathrm{A}$ ne dépend pas de $\mathrm{A}$ directement sans quoi les dogmes qui formulent des mystères ne pourraient pas être crus. Car si on veut leur substituer des Images, du non-langage, on ne le peut.

Il faut noter qu'ici la production d'images est le fait de l'imagination, c'est-à-dire de la capacité à se re-présenter en "images" ce qui a été vécu dans l'expérience sensible (nous retrouvons ici les nominalistes empiristes du débutde l'exposé)à ne surtout pas confondre avec l'imaginaire - fonction négative par excellence pour Valéry, car c'est lui qui permet la production du fiduciaire, c'est-à-dire du fictif, de la croyance qui n'est fondée sur aucune encaisse véritable.

C'est bien ce qu'il dénonce en poursuivant le démontage du mécanisme de la croyance :

Il faut donc et il suffit que des formules sans signification exhaustive produisent des effets ou valeurs d'action ou d'attitude. Mais alors ce sont de simples signaux - et on pourrait leur substituer d'autres formules qui forment les mêmes effets. On peut inculquer d'autres dogmes et nommer d'autres mystères. En résumé, croire (au sens des religieux) c'est ne pas réagir à l'arbitraire des formules.

Et pour Valéry, les sociétés - peut-être parce que sociétés - sont fondées sur la mentalité mythique dont l'esprit religieux n'est qu'une des formes. Pourtant comme nous l'avons dit, il croit au pouvoir de l'Esprit - et à la force désaliénante d'une analyse critique des systèmes de signes. Aussi peut-il intituler ironiquement une page d'un Cahier de 1942 (C. XXVI, 231-232) Credo, puisqu' en fait il s'agit d'un retournement subversif des systèmes de croyances héritées - un credo 
articulé sur un possum - après étude de la parole - (notez bien que pour Valéry, la seule parole "vraie" est celle qui s'inscrit dans l'acte d'énonciation, dans une présence dans la voix, à partir du corps, dans une relation donc vécue entre C.E.M. (avec leurs diverses combinatoires) ou d'une Parole ayant son répondant en acte dans le réel.

Credo.

Je crois à la dissolution, disparition ou transmutation assez prochaine de ces grandes mythologies connues sous les noms de Philosophie et d' Histoire.

"Mythologies", c’est-à-dire Créations du Crédit, c'est-à-dire du langage.

C'est pourquoi Philosophie et Histoire seront plus ou moins remplacées par l'étude des valeur de la parole - Etude qui classera les œuvres de ces espèces entre le roman et les poésies - sans oublier les Livres Saints, la Théologie etc... - toute la bibliothèque de la Fiducia.

Sc En regard, le stock scientifique

- composé :

$1 \%$ du stock toujours croissant

- a/ des observations enregistrées que l'on peut refaire ausssi souvent qu'on veut

(du moins à l'état d'enregistrements matériels)

- b/ des recettes précises -Fac secundum artem. (Acta -agenda) $2^{\circ}$ : de l' amas des nébuleuses... je veux dire des théories - à l'état ou d'instruments ou d'œuvres d'art, ou de documents - (verba) Ainsi, dans l'ensemble, c'est le Faire qui l'emporte... Tant pis ! peut-être - (1942 - C. XXVI, 231-232).

Valéry semble faire le bilan de son entreprise de mythoclaste avec un certain regret d'être parvenu à ses fins. En effet, démystifiant même les grandes créations de l'Imaginaire, il ne leur confère plus aucune valeur, sinon celle d'être de "beaux objets verbaux" à contempler 
- mais qui ne se donnant pas comme tels peuvent poursuivre pour d'autres que lui leur emprise mystificatrice. Résolument matérialiste, Valéry a défini les limites de son entreprise : épuiser le champ des possibles dans les limites de la vie d'un homme, qui est - ne l'oublions pas - mortel, et de ses pouvoirs "réels", non imaginaires. Seul le stock "scientifique" permettant la domination physique et matérielle du monde d'une part, et le travail d' analyse critique d'autre part auraient-ils une "valeur" réelle pour Valéry?

Voici un extrait révélateur de ce qui me paraît le "générateur" de la démarche valéryenne, que j'emprunte encore au Cahier 240, dans la mesure où les Cahiers écrits durant les dernières années de sa vie sont souvent des bilans, des retours sur son questionnement :

que peut...?

Ce que je puis? Ce que je ne puis?

Voilà la grande affaire.

Et que vaut ce que je puis?

Toute la philosophie se réduit à exprimer nettement

$1^{\circ}$ ce possum

Je puisquand

comment, moyennant quoi

dans quel état

sous quelle excitation etc.

réside

dans le domaine

moteur-direct

et indirect-arrêt-

action volontaire et assimilée $2^{\circ}$ ce qu'il vaut

faire $>$ façonner

$>$ provoquer

$>$ détruire, annuler

$>$ empêcher

>imiter-répéter-reproduire

$>$ prévoir

$>$ conserver-durer

$>$ distinguer-

Valéry réunit sous le signe du "possum facere" deux affixes pour synthétiser les possibles du passage à l'acte - pierre de touche du réel - BLE (faisable, etc...) et RE (répéter-reproduire). Mais pour "réduire" 
le possum - c'est-à-dire trouver la réponse à la question "que peut un homme ?", il y a cette entreprise d'observation-réflexion sur le(s) langage(s) - qui aboutit à une véritable entreprise de démystification, à partir de laquelle pourra se fonderun véritable "possum" et l'évaluation de la valeur de celui-ci. Nous allons rencontrer ici un des "nœuds" de croisement possible avec la réflexion socio-critique.

On peut dire que le développement des pouvoirs conscients de l'Homme, c'est-à-dire d'un $M o i$, permettra la mise en jeu d'une analyse, qu'on peut qualifier de "sémiotique", ouvrant à une distance critique, en fait une désaliénation - c'est l' aspect critique - , mais aussi à la possibilité d'une "praxis créatrice" qui se situera sur deux plans : celui de la science et celui de l'esthétique.

Dans un premier temps Valéry entreprend la démystification de tous les discours fiduciaires qui recouvrent à peu près toutes les pratiques socio-culturelles car toutes intimément liées à la langue héritée, c'est-à-dire à toutes les strates des diverses paroles qui se sont réifiés pour devenir "choses mortes" - coupées du réel - c'est-à-dire de l'articulation C-E-M où le langage pourrait s'originer à nouveau à partir du geste, de l'acte, du pouvoir de l'énonciation s'inscrivant dans un ici et un maintenant. Quelle est la situation de Valéry dans le langage hérité ?: "Nous pensons, nous écrivons dans un langage auquel nous ne croyons plus".

Valéry reconnaît ici, quoiqu'il en ait -il a rêvé d'un "langage-self", il a tenté d'en approcher; mais peut-il y avoir un langage singulier? -, qu'il continue de fonctionner dans le cadre social global qui l'entraîne, et que la fiducia formant la plus grande partie du langage fait aussi partie du fonctionnement du moi. Et Valéry continue :

Nous savons qu'il emporte avec lui une pluralité désordonnée de conceptions du monde, d' hypothèses physiques, cosmologiques, psychologiques, qui ont fait les philosophes se rompre inutilement la tête au sujet d'êtres et d'essences qui n'existent pas ; comme l'être, l'âme, le temps, la volonté, etc. 
Mais comment en construire un autre, et comment le concevoir ? (C. I, 411 - C. VIII, 141-142).

Valéry va établir une échelle des signes : leur valeur sera à la mesure de leur pouvoir. Les signes les meilleurs sont ceux qui ont un pouvoir de domination physique et matérielle du monde. Les autres sont de l'ordre du fiduciaire, du mythique : une monnaie dévaluée, sans fondement réel - sur laquelle la société vit à crédit, société dans laquelle les individus ne savent pas qu'ils sont victimes de croyances, qu'ils sont manipulés. Le fiduciaire pour Valéry est tout acte phénoménal ou mental qui s'établit sur un contrat de convention entre l'instance émettrice et l'instance réceptrice. Il s'établit dans un système d'échanges (qui peut servir de modèle à la communication mais que Valéry aligne sur le modèle économique), avec lequel il est en relation. Valéry dépsychologise la linguistique - et l'ensemble des productions culturelles, l'ensemble des pratiques sociales, qui fonctionnent comme des "langages", et rapproche cet ensemble qu'on pourrait appeler "symbolique" de l'économie. Il peut alors affirmer : "La société est le fonctionnement de deux systèmes de signes : le langage et la monnaie".

En fait l'entreprise de démythification de Valéry, parce qu'elle s'est située à deux niveaux va permettre de redonner une "valeur" à certains discours qu'il avait classés dans la Bibliothèque de la Fiducia. Comment? En coupant le discours mythique de ses prolongements dans l'imaginaire qui donne un sens fictif aux signes et qui fait croire à la signification du Monde en construisant un pseudo-réel, il va extraire le discours poétique - et les discours esthétiques plus généralement de la Bibliothèque de la Fiduciaet le rétablir dans sa vraie fonctionnalité. Pour fonder la validité du langage poétique, il ne l'inscrit pas comme la science ou les conduites pragmatiques dans la perspective d'une finalité opératoire sur les choses. Valéry ne l'inscrit pas non plus dans une tradition de la modernité qui, établissant un contre-discours par rapport à l'arbitraire du langage, pense que proférer c'est créer de l'Etre - renouant ainsi avec le néo-platonisme d'un Plotin - ; pour Valéry il s'agit d'une 
croyance mythologique. Lui s'attache au sujet producteur qui opère sclon le système C-E dans sa relation au Monde. La "fabrication de l'œuvre" est la preuve d'un esprit qui retrace l'itinéraire de son aventure charnelle dans et par une Forme. Valéry a une certitude sensible - qu'il a construite - "son" corps. Mais le corps ne pense pas. Alors, par un mouvement dialectique, l'esprit reconstruit, conquiert à nouveau, et symboliquement, la structure enfouie dans le labyrinthe charnel, comme le souligne Patricia Signorile dans ses travaux sur Valéry et l'Architecture. Il peut alors, paradoxalement, "écrire de tout son corps". Et La Jeune Parque peut être une autobiographie du Moi (Corps-Esprit dans son rapport au Monde) parce qu'elle ne prend pas la forme d'un récit qui en donnerait une transposition pseudo-réelle, faisant confiance à la transitivité référentielle du langage, à la pseudo-mimésis, mais qu'elle est avant tout Forme homologique de cette relation. La Poésie sort ainsi des formes fiduciaires illusoires qui reposent sur la Mimésis, comme le Roman ou l'Histoire. Le travail du poète portera avant tout sur le langage comme matériau sensible. La valeur du poème viendra de l'ensemble des modifications actives touchant la sensibilité du récepteur.

Art de la Parole, le matériau sensible du poète seront les mots, mais portés par le Rythme et la Voix. Il y faudra d'abord une sorte de régression dans l'expérience sensible infra-nominale où se trouve la source du langage. La valeur du poème se mesurera donc à son effet "réel", à savoir à sa capacité à faire éprouver un maximum de jouissance dans un échange énergétique, et non à des pouvoirs leurrants portant sur l'imaginaire. Ce maximum de jouissance, c'est l'Infini esthétique selon Valéry, un infini qui s'inscrit dans une forme finie. Pourquoi ? Parce que seule la forme finie est en homologie avec le réel :

Toute ma philosophie est dominée par l'observation du caractère fini par raison fonctionnelle de toute "connaissance". Ce caractère est réel tandis que le non-fini est fiduciaire. 
Le mot "infini" est alors débarrassé de ses connotations mystiques, vagues, fiduciaires. Il faut le comprendre selon le modèle suivant :

\section{Infini Esthétique !}

L'Enfant dit : Encore, c'est son jugement esthétique. (C. XVIII, 208).

Valéry peut redonner de la valeurau Poème, et plus généralement à l'œuvre d'art en plaçant l'œuvre dans la relation production-réception. Il n'y a plus d'œuvre d'art en soi : l'œuvre est production d'un sujet reçue par un autre sujet. Il n'est plus possible de construire une image de l'objet esthétique, mais seulement des rapports que nous entretenons avec lui :

L'excitation que nous donnent certains objets - (...) l'espèce d'infini que j'ai appelé esthétique - et qui consiste dans la reprise (du désir) résultant de la possession - nous fait désirer en outre de l'engendrer et provoquer ad libitum - et c'est ici un infini de second degré (1934 - C. XVIII, 457).

Mais la valeur fonctionnelle donnée à la création esthétique (production-réception) ne se limite pas au fait que celle-ci tend à produire le maximum de jouissance. Ces effets peuvent atteindre au maximum d'intelligibilité, car dans l'œuvre telle qu'elle apparaît pour Valéry et chez Valéry se retrouvent les trois dimensions de la connaissance, selon l'articulation C-E-M :

Les trois dimensions de la connaissance : Le corps, l'esprit, le monde.

Cette division simpliste est pourtant capitale. Elle est cachée dans toute connaissance.

L'homme qui s'éveille retrouve ces 3 groupes.

Le corps comprend les forces. 
Quand on dit : Cet arbre - Le tout s'adresse à l'esprit. Arbre est du monde. Cet est du corps - axe de référence.

Au point de vue accidentel - le corps est monde.

L'action / effet / du monde sur le corps est esprit - (sensation question). L'action / effet / de l'esprit sur le monde est acte.

La pluralité des rôles, des symboles de notre corps.

Serviteur, maître - une partie est notre esclave, et le tout est notre maître (C. VIII, 203).

L'œuvre d'art pour Valéry doit restituer au destinataire, sous forme énergétique, l'état unitif du corps et de l'esprit. Chaque forme d'art doit être informée par le matériau sensible, car c'est la forme qui fait sens, à condition de ne pas oublier le sensible - le lien étant produit par l'imagination (qui s'oppose toujours chez Valéry à l'imaginaire) qui est l'expérience vécue par le corps revenant dans l'esprit.

L'analyse de la pensée esthétique en relation avec l'analyse de son moi ont permis à Valéry de saisir l'homologie qui existe entre la vie perceptive et la vie pensante, sans toutefois réduire la seconde à la première. Dans ses Contes ou Poèmes abstraits, dans ses projets dramatiques, Valéry, déjouant les risques d'une écriture mimétique,peut tenter de construire des œuvres jouant sur des mythes dé-théologisés, car ayant pour seul statut celui de "place" dans le jeu opératoire du fonctionnement de l'esprit. L'Artiste-Penseur a encore un rôle dans les limites du sujet même.

Dans le dernier grand projet qui a été le sien, celui du IIIème Faust, Valéry tente de créer le Mythe du Mythoclaste. Le projet théâtral de Mon Faust c'est de mettre en scène un mythe qui dise : "Voici nos mythes, nos erreurs que nous avons eu tant de peine à dresser contre les précédent !..." (E. II, 626), phrase quel'on trouve dans Tel Quel (1926) mais qui reprend, en la modifiant légèrement, une phrase de 1904, extraite des Cahiers $(C$. III, 267) : "Voici nos mythes que tant de peine nous eûmes à élever contre de plus anciens".

Entreprise singulière d'un Sujet qui tente de créer une œuvre critique qui soit aussi Forme - dans laquelle se conjuguent la critique 
socio-culturelle du discours fiduciaire, la sémiotique du langage intérieur, avec ses possibilités d' action sur le réel problématisé... et ses harmoniques : Faust figure l'idée de pouvoir réel d'action subsistant, mais ce pouvoir lui-même se trouve incertain dans son emploi. Méphistophélès synthétise en lui les sentiments, les instincts, l'art de se tromper soi-même et autrui. Les harmoniques figurées par le Solitaire et les Fées,

ce sont les valeurs supérieures de la sensibilité, qui s'ordonnent en groupes (au sens quasi-mathématique du mot) et qui sont la structure abstraite de nos modifications les plus concrètes - les sensations en soi, au-dessus de toute signification et au-dessus de toute condition accidentelle de leur production fragmentaire. C'est l'Art qui a pour fonction de révéler ces groupes (1942- $C$. T. I, 306 - C. XXIV, 440-442).

Le travail critique de Valéry articulé sur sa recherche du fonctionnement de l'esprit et des pouvoirs du sujet lui a permis de tenter de fonder une œuvre qui ne soit pas une réponse mystificatrice à des questions sans réponses, mais invention - c'est-à-dire découverte - de la structure dynamique de la sensibilité dans une relation dedans/ dehors, concret/abstrait ; seule maîtrise possible d'un rapport à un monde sans signification, mais dont la mise en forme fait sens - et que Valéry, comme L'Ange de son poème, "ne cessa de connaître et de ne pas comprendre" - à partir d'un Moi qui est :

le rôle plus ou moins caché du corps vrai dans la conscience. Corps vrai - c'est-à-dire non le corps visible et imaginable, l'anatomique, mais l'intime travail et fonctionnement qui est véritablement notre corps, notre facteur. Notre corps dont la matière se renouvelle continuellement est donc comparable à une formule (chimico-énergétique) de formules. Formule d'équilibre mobile, où le stable résulte des instables (1921-1922, C. VIII, 497). 
L'œuvre de Paul Valéry a par là, en même temps que l'Infini esthétique qu'il peut procurer, valeur d'excitant pour des recherches à venir. 